\title{
Response-force manipulations in fixed-ratio schedules
}

\author{
EDWARD K. CROSSMAN and RICHARD W. SERNA \\ Utah State University, Logan, Utah 84322
}

\begin{abstract}
Response force (effort) was manipulated by varying the required travel of a pigeon's pecking key. Performances under a fixed-ratio 2 schedule and a fixed-ratio 20 schedule were compared at the different forces. Results indicated that increasing the force requirement produced a lower overall response rate through a lengthening of the preratio pause; at fixed ratio 2, the interresponse times were also lengthened slightly, but not at fixed ratio 20.
\end{abstract}

Interest by psychologists in the "law of least effort" as a basic principle underlying animal behavior became evident during the late 1920s and 1930s (Keller \& Schoenfeld, 1950). The length and structure of alleys in mazes (Solomon, 1948), the force required to press a lever (Skinner, 1938), and so on, were popular variables singled out for manipulation. By 1943, Hull recognized the importance of effort, or force, as an inhibitory factor to be subtracted from the reaction potential (sEr) produced by other, noninhibitory variables. In contrast, operant conditioners have chosen to emphasize variations of the law of effect; only scant, sporadic attention has been given to response effort. Perhaps, in large measure, this lack of attention can be traced to the popularity of pigeon subjects and the predominance of a low-force manipulandum, the pigeon pecking key.

The exclusion has not been total, however. For example, Notterman and Mintz (1965) have summarized a number of studies on response topography, including response force. Chung (1965), in a series of carefully conducted experiments, demonstrated that in both simple variable-interval (VI) and concurrent (conc) VI schedules, the behavior of pigeons was sensitive to pecking-force variations. In an attempt to produce a pecking key with force-time characteristics that are more stable than those associated with the common, springloaded pecking keys, Rilling, Kramer, and Askew (1970) designed a nonmovable, plastic pecking key with embedded strain gauges to record strike force. Their key had problems associated with its resonant frequency, however, and currently the spring-loaded, microswitchactivated pecking key continues unabated as the most popular transducer in pigeon studies. Very recently, Hunter and Davison (1982) investigated key force and reinforcement rate on conc VI.VI schedule performance. Unlike Chung, these investigators found that responseforce requirements did not interact with reinforcement rate in affecting preference; increasing force on one of two keys did, however, affect response rate on that key.

It seems apparent, then, that while response effort has demonstrable effects on performance, the paucity of research in this area leaves us with only a vague under- standing of this variable. The present study was conducted to examine the fine-grain temporal characteristics of simple fixed-ratio (FR) schedule performance under two different levels of key force. Unlike the previous studies, which manipulated force by adjusting either spring tension (Chung, 1965) or the mass of the pecking key (Hunter \& Davison, 1982), the current investigation manipulated key throw, or the distance that the key could travel; according to Hooke's law, force is directly proportional to distance $(\mathrm{F}=\mathrm{kd})$.

\section{METHOD}

\section{Subjects}

Four experimentally naive pigeons of mixed breed served. The subjects were maintained at $80 \% \pm 15 \mathrm{~g}$ of their free-feeding body weights. They had free access to water and were occasionally fed, in their home cages, to maintain their $80 \%$ weights.

\section{Apparatus}

All experimental events were controlled by a PET 2001 Commodore microcomputer (Crossman, Stephenson, \& Lynch, 1980 ) interfaced with electromechanical equipment housed in a room separate from that which housed the experimental chambers. The same equipment also recorded the data on cassette tape for later analysis by a similar computer system. The experimental chambers, $53.3 \mathrm{~cm}$ high, $61 \mathrm{~cm}$ deep, and $40.6 \mathrm{~cm}$ wide, were identical except for the length of the key throw. The chambers contained three $2.5-\mathrm{cm}$ plastic keys (see Figure 1) $5.1 \mathrm{~cm}$ apart, $25.4 \mathrm{~cm}$ above the floor. Only the left key was used and was at times illuminated with a red hue. In one chamber (short-key chamber), the length of the key throw, or length of movement of the plastic key before the microswitch would close, was $.76 \mathrm{~mm}$. In the other chamber (long-key chamber), the throw measured $4.32 \mathrm{~mm}$. Key throw was altered by changing the distance between the key and the microswitch. A food hopper containing Purina pigeon checkers was situated directly below the center key and $6.4 \mathrm{~cm}$ above the floor of the chamber. At reinforcement, the hopper was lifted for $3 \mathrm{sec}$, during which time the hopper light was illuminated and the key light was extinguished. The houselight remained on during the entire session. Pecks to the darkened keys had no scheduled consequence. Each session terminated in blackout when 30 reinforcers had been delivered. White noise attenuated extraneous sounds.

\section{Procedure}

All subjects (B400, B401, B402, B403) were initially hand shaped to approach the food hopper and peck the red key for 


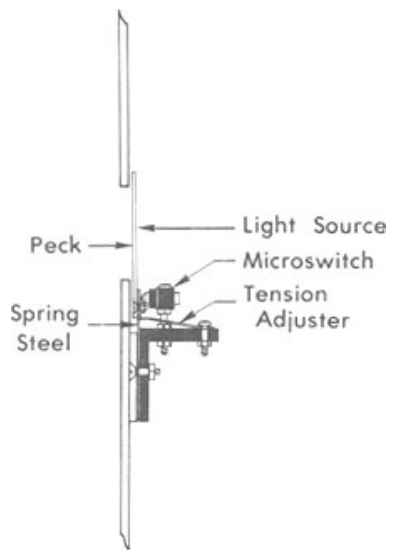

Figure 1. Diagram of pigeon pecking key.

continuous reinforcement. Table 1 shows the number of sessions for each subject in each condition and the order of exposure to each condition. Subjects initially shaped in the long-key chamber (B400 and B401) for 1 day were then placed on an FR 2 schedule in the same chamber. Subjects B402 and B403, shaped in the short-key chamber, were also placed on an FR 2 in the short-key chamber. When the subjects' behavior was stable, they were switched to the opposite chamber while the same schedule remained in effect. All subjects were then changed to FR 20 in the same chamber. Following this, the subjects were again switched to the opposite chamber while the same schedule remained in effect. Thus, a comparison of the effects of different length key throws and schedules could be made.

In all conditions, the number of responses emitted, preratio pauses (PRPs), and interresponse times (IRTs) were recorded. Stability was defined as the lack of a consistent trend in either the mean PRP or the mean IRT for the last five sessions of a condition.

\section{RESULTS}

Basically, two dependent variables were examined in the present experiment: (1) the length of pause, or latency, prior to the first response in a ratio (PRP) and (2) the amount of time between successive responses (IRT). Figure 2 shows the relation among key throw, FR schedule, and the PRP. The data represent the means of the last five sessions under a condition; standard deviations are also indicated (see vertical lines inside each bar). For all four subjects, the PRP was longer at FR 20 than at FR 2, regardless of key throw. However, for B402, the effect was slight.
Key throw also affected the PRP. Typically, at both FR 2 and FR 20, the PRP was longer when the key throw was long than when it was short. Again, this effect was only slight for B402, and a reversal can be seen in the data for B403 at FR20.

Data are presented in Figure 3 that show how key throw affected IRTs for all four birds. Again, the data in this figure are means from the last five sessions at each condition; standard deviations are also shown, as in Figure 2. FR size did not affect mean IRT (see Figure 3 ) in a consistent way. Birds B400 and B401 tended to increase their mean IRTs at FR 20, but this was not true for B402 or B403. Key throw did appear to affect IRT length at FR 2. For all birds, except B403, the mean IRT was longer at FR 2 when the key throw was long. At FR 20, however, there was no relationship between key throw and IRT length.

Several additional temporal variables were examined: IRTs by position in FR, PRPs by position in session, and IRT sequential dependencies. IRTs by position in FR refers to a comparison of IRTs that occurred early in a particular FR with IRTs at the end of the FR. In the measure PRPs by position in session, the lengths of PRPs within a session were examined to determine whether any correlation might exist between PRP length and when within a session the PRP occurred (e.g., early vs. late). No consistent relations were noted in either of these two measures. IRT sequential dependencies constitute a measure that compares the lengths

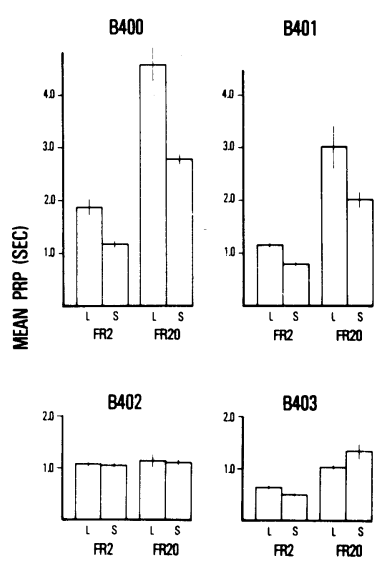

Figure 2. Mean preratio pauses (PRPs) as a function of key throw. Data are from last five sessions.

Table 1

Sequence of Conditions for B400, B401, B402, and B403

\begin{tabular}{|c|c|c|c|c|c|c|c|c|c|}
\hline \multirow[b]{2}{*}{ Condition } & \multirow[b]{2}{*}{ Schedule } & \multirow[b]{2}{*}{ Key Throw } & \multicolumn{2}{|c|}{ Sessions } & \multirow[b]{2}{*}{ Condition } & \multirow[b]{2}{*}{ Schedule } & \multirow[b]{2}{*}{ Key Throw } & \multicolumn{2}{|c|}{ Sessions } \\
\hline & & & B400 & B401 & & & & B402 & B403 \\
\hline 1 & FR 2 & Long & 15 & 14 & 1 & FR 2 & Short & 15 & 24 \\
\hline 2 & FR 2 & Short & 14 & 16 & 2 & FR 2 & Long & 13 & 14 \\
\hline 3 & FR 20 & Short & 14 & 14 & 3 & FR 20 & Long & 14 & 15 \\
\hline 4 & FR 20 & Long & 15 & 17 & 4 & FR 20 & Short & 14 & 16 \\
\hline
\end{tabular}



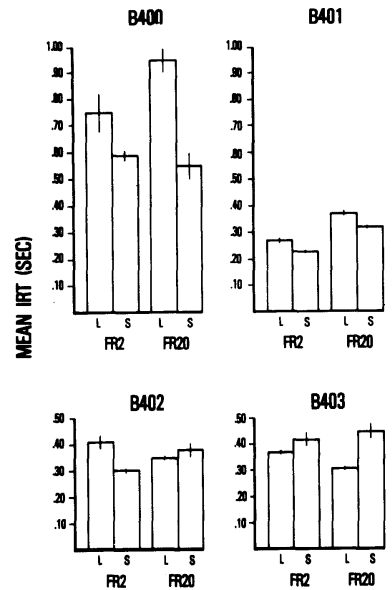

Figure 3. Mean interresponse times (IRTs) as a function of key throw. Data are from last five sessions.

of pairs of adjacent IRTs throughout the ratio. Thus, the first IRT is compared to the second, the second to the third, and so on. During each session, IRTs were first categorized as short, medium, or long (the IRT distribution was divided into thirds). Next, the lengths of adjacent pairs of IRTs were compared, and the outcome incremented one of the nine possible categories, such as short-short, short-medium, and so on. This measure revealed that under all conditions for all birds, the largest category was short-short. That is, under FR 20 (FR 2 was excluded since it has only one IRT), if a short IRT had just occurred, it tended to be followed by another short IRT. A similar finding has been reported elsewhere for both small and large FR schedules (Crossman, Bonem, Bonem, Green, \& Trapp, Note 1). No other consistencies were observed in this measure, nor did key throw seem to affect it.

\section{DISCUSSION}

The present experiment demonstrated that when force was manipulated by altering key throw, reliable behavioral effects were produced. The most notable effect was a lengthening of the mean PRP as required key throw was increased. At FR 2, the mean IRT was similarly lengthened, but not at FR 20 .

An increase in PRP amounts to a reduction in overall response rate on the FR schedule. Consonant with this result, two other studies have reported response rate reductions dependent upon an increase in required key force (Chung, 1965; Hunter \&
Davison, 1982). These studies altered force by changing either spring tension or the mass of the key, rather than the required distance of travel. Nevertheless, the similarity in findings suggests that a common variable (i.e., force) had in fact been manipulated.

As Chung (1965) stated, the response rate reductions could simply be an artifact of manipulating the force variable. Thus, increasing force requirements might not alter respone rate per se; rather, a larger proportion of pecks at the higher forces go unrecorded because they fail to meet the force criterion. Chung presented evidence that the response rate reduction that he observed resulted, at least in part, from a slower emission of criterion keypecks. A similar conclusion can be derived from the IRT data in the present experiment. If a higher key force increased the proportion of subcriterion responses, then the time between responses should have increased, as did the PRP. The mean IRT did increase slightly at FR 2, but not at FR 20, under which the larger increases in PRP occurred when key throw was increased. Therefore, force, or effort, the physiological derivative, would seem to fall into the same class as other inhibitors such as punishment, in that these variables are inversely related to response rate.

\section{REFERENCE NOTE}

1. Crossman, E. K., Bonem, E. J., Bonem, M. K., Green, G. M., \& Trapp, N. L. Development of temporal patterns in small FRs: $A$ microanalysis. Paper presented at the Association of Behavior Analysis, Milwaukee, 1982.

\section{REFERENCES}

Chung, S. H. Effects of effort on response rate. Journal of the Experimental Analysis of Behavior, 1965, 8, 1-8.

Crossman, E. K., Stephenson, M. R., \& LYnCh, H. L. An intelligent PET interface for environmental control and data acquisition and analysis. Behavior Research Methods \& Instrumentation, 1980, 12, 238-240.

Hunter, I., \& DAvison, M. Independence of response force and reinforcement rate on concurrent variable-interval schedule performance. Journal of the Experimental Analysis of Behavior, 1982, 37, 183-187.

Keller, F. S., \& Schoenfeld, W. N. Principles of psychology. New York: Appleton-Century-Crofts, 1950.

Notterman, J. M., \& Mintz, D. E. Dynamics of response. New York: Wiley, 1965.

Rilling, M., Kramer, T. J., \& Askew, H. R. A preliminary analysis of the dynamics of the pecking response in pigeons. Journal of the Experimental Analysis of Behavior, 1970, 13, 267-278.

Skinner, B. F. Behavior of organisms. New York: AppletonCentury-Crofts, 1938.

Solomon, R. L. The influence of work on behavior. Psychological Bulletin, 1948, 45, 1-40.

(Received for publication October 15, 1982.) 